\title{
Patient outcomes after laminotomy, hemilaminectomy, laminectomy and laminectomy with instrumented fusion for spinal canal stenosis: a propensity score-based study from the Spine Tango registry
}

\author{
Everard Munting $\cdot$ Christoph Röder • \\ Rolf Sobottke - Daniel Dietrich · Emin Aghayev • \\ on behalf of the Spine Tango Contributors
}

Received: 12 November 2013/Revised: 26 April 2014/ Accepted: 26 April 2014/Published online: 20 May 2014

(c) Springer-Verlag Berlin Heidelberg 2014

\begin{abstract}
Purpose To compare patient outcomes and complication rates after different decompression techniques or instrumented fusion (IF) in lumbar spinal stenosis (LSS).

Methods The multicentre study was based on Spine Tango data. Inclusion criteria were LSS with a posterior decompression and pre- and postoperative COMI assessment between 3 and 24 months. 1,176 cases were assigned to four groups: (1) laminotomy $(n=642)$, (2) hemilaminectomy $(n=196)$, (3) laminectomy $(n=230)$ and (4) laminectomy combined with an IF $(n=108)$. Clinical outcomes were achievement of minimum relevant change in COMI back and leg pain and COMI score (2.2 points), surgical and general complications, measures taken due to complications, and reintervention on the index level based on patient information. The inverse propensity score weighting method was used for adjustment.

Results Laminotomy, hemilaminectomy and laminectomy were significantly less beneficial than laminectomy in combination with IF regarding leg pain (ORs with $95 \%$ CI $0.52, \quad 0.34-0.81 ; \quad 0.25, \quad 0.15-0.41 ; \quad 0.44, \quad 0.27-0.72$,
\end{abstract}

\section{E. Munting}

Clinique Saint Pierre, Avenue Reine Fabiola 9,

1340 Ottignies, Belgium

C. Röder · D. Dietrich · E. Aghayev $(\bowtie)$

Institute for Evaluative Research in Medicine, University of

Bern, Stauffacherstrasse 78, 3014 Bern, Switzerland

e-mail: emin.aghayev@memcenter.unibe.ch

R. Sobottke

Department of Orthopaedic Surgery, Medizinisches Zentrum

Städte Region Aachen, Mauerfeldchen 25,

52146 Würselen, Germany respectively) and COMI score improvement (ORs with $95 \%$ CI 0.51, 0.33-0.81; 0.30, 0.18-0.51; 0.48, 0.29-0.79, respectively). However, the sole decompressions caused significantly fewer surgical (ORs with $95 \%$ CI 0.42 , $0.26-0.69 ; 0.33,0.17-0.63 ; 0.39,0.21-0.71$, respectively) and general complications (ORs with $95 \%$ CI 0.11 , $0.04-0.29 ; 0.03,0.003-0.41 ; 0.25,0.09-0.71$, respectively) than laminectomy in combination with IF. Accordingly, the likelihood of required measures was also significantly lower after laminotomy (OR $0.28,95 \%$ CI $0.17-0.46$ ), hemilaminectomy (OR 0.28, $95 \%$ CI 0.15-0.53) and after laminectomy (OR 0.39, $95 \%$ CI 0.22-0.68) in comparison with laminectomy with IF. The likelihood of a reintervention was not significantly different between the treatment groups.

Discussion As already demonstrated in the literature, decompression in patients with LSS is a very effective treatment. Despite better patient outcomes after laminectomy in combination with IF, caution is advised due to higher rates of surgical and general complications and consequent required measures. Based on the current study, laminotomy or laminectomy, rather than hemilaminectomy, is recommendable for minimum relevant pain relief.

Keywords Lumbar spinal stenosis - Decompression . Instrumented fusion $\cdot$ Spine Tango $\cdot$ Propensity score

\section{Introduction}

The incidence of lumbar spinal stenosis (LSS) has been estimated between 3.9 and $11 \%$ of the population $[1,2]$ and its prevalence is steadily growing in our ageing society [3]. Decompressive surgical treatment for carefully 
selected patients with LSS has been shown to have greater efficacy in comparison with nonsurgical treatments [4-7].

Today, various decompressive surgical procedures differing in the invasiveness of the intervention exist. They range from minimally invasive segmental laminotomy preserving all posterior structures to large laminectomy with facetectomy. The most frequently performed interventions are decompression using laminotomy, laminectomy and decompression combined with an instrumented fusion (IF) of the treated segment. If a pathologic or iatrogenic destabilization of the spine is suspected, a more radical and invasive approach like an IF is usually chosen [8].

Use of one or the other decompression technique is decided upon at the discretion of the clinician. A wide variability in surgical decision making for patients with LSS and a lack of consensus is a matter of fact [9-11]. Numerous measurable and unmeasurable factors may influence the surgeon's decision about his decompression technique, with or without an IF. The comparative effectiveness and complications of various decompressive procedures are uncertain $[8,12]$.

The international Spine Tango registry, administered under the auspices of EuroSpine, The Spine Society of Europe and hosted at the Institute for Evaluative Research in Orthopaedic Surgery at the University of Bern in Switzerland, documents a wide range of spinal surgical procedures in combination with patient-related outcomes [13]. Currently, over 70 hospitals from 16 European and nonEuropean countries voluntarily contribute their patient data to the registry along with data on treatment procedures for LSS [13].

Comparative treatment assessments would ideally be done based on randomized treatment assignment to balance the baseline patient characteristics. This, however, is not always feasible in real-life medical practice due to a number of ethical, administrative, financial, and numerous other considerations. On the other hand, there are statistical methods used in observational studies to reduce selection bias and confounding caused by a non-random treatment assignment. One technique is the propensity score [14]. Although the methodology was first described in the 1980s, it has only recently gained popularity and entered orthopaedics [15]. This analytic approach produces a single variable-a propensity score-from numerous covariates simplifying therewith the adjusted comparison. The propensity score allows for comparison of patients with similar characteristics between the studied groups and consequently enables investigators to adjust for selection bias due to measured factors, though it still cannot account for selection bias that is due to unobserved factors.

Using data of the international Spine Tango registry, the comparative effectiveness of three different decompression types and decompression in combination with an IF was
Table 1 Types of instrumented fusion

\begin{tabular}{|c|c|}
\hline Type of instrumented fusion & $\begin{array}{l}\text { Laminectomy }+\mathrm{IF} \\
n=108\end{array}$ \\
\hline Pedicle screws with rod & $49(45.37 \%)$ \\
\hline $\begin{array}{l}\text { Pedicle screws with rod }+ \text { interbody } \\
\text { stabilization with cage via posterior } \\
\text { approach }\end{array}$ & $47(43.52 \%)$ \\
\hline $\begin{array}{l}\text { Pedicle screws with rod }+ \text { "other" type of } \\
\text { instrumentation }\end{array}$ & $2(1.85 \%)$ \\
\hline Pedicle screws with rod + facet screws & $2(1.85 \%)$ \\
\hline $\begin{array}{l}\text { Interbody stabilization with cage via posterior } \\
\text { approach }\end{array}$ & $2(1.85 \%)$ \\
\hline $\begin{array}{l}\text { Pedicle screws with rod }+ \text { transarticular } \\
\text { screws }\end{array}$ & $1(0.93 \%)$ \\
\hline $\begin{array}{l}\text { Pedicle screws with rod }+ \text { interbody } \\
\text { stabilization with cage via posterior } \\
\text { approach }+ \text { pedicle screws with plate }\end{array}$ & $1(0.93 \%)$ \\
\hline $\begin{array}{l}\text { Interbody stabilization with cage via posterior } \\
\text { approach }+ \text { laminar hooks with rod }\end{array}$ & $1(0.93 \%)$ \\
\hline $\begin{array}{l}\text { Interbody stabilization with cage via posterior } \\
\text { approach }+ \text { pedicle screws and plate }\end{array}$ & $1(0.93 \%)$ \\
\hline $\begin{array}{l}\text { Pedicle screws with plate }+ \text { transarticular } \\
\text { screw }\end{array}$ & $1(0.93 \%)$ \\
\hline "Other" type of instrumentation & $1(0.93 \%)$ \\
\hline
\end{tabular}

$I F$ instrumented fusion

analysed. An adjusted analysis based on propensity scores was applied in searching for predictors of the desired outcome.

\section{Materials and methods}

The registry

'Surgery 2006' is a physician-based form in the registry containing epidemiological and diagnostic data, treatment parameters, description of intraoperative surgical and general complications, length of hospital stay, etc. The register allows linking these data to outcome data. Beyond the surgical records, patients are asked to complete a selfreported Core Outcome Measure Index (COMI) questionnaire with two graphical pain scales (GPS 0-10 points) for back and leg pain [16]. The instrument documents five domains (pain, back-related function, symptom-specific well-being, general quality of life and disability (social and work)), based on which a COMI score is calculated. Follow-up COMIs also include a question on reintervention "Since the operation in our hospital, have you had any further operation(s) on your lumbar spine in our or in other hospitals?" with three possible answers: "no", "yes, but at a different level of the spine", "yes, at the same index level of the spine". 
Patient sample

The database of the registry was screened on November 21st 2012 for the diagnosis of degenerative LSS treated with laminotomy, hemilaminectomy, laminectomy, or laminectomy in combination with an IF. Degenerative spondylolisthesis grades 0-1 were allowed. A total of 6,752 cases were identified. Patients with additional percutaneous or motion-preserving measures, patients with non-degenerative spondylolisthesis and patients with 'unknown' ASA status ( $n=1,009$ ) were excluded. Patients without information on ASA status or patients with ASA4 or ASA5 statuses were also excluded for reasons of sample homogeneity and adjustment accuracy $(n=44)$. Of the remaining 5,699 patients, 1,176 had completed a baseline and at least one follow-up COMI assessment between 3 and 24 months postoperatively. The 1,176 patients were assigned to four groups: (1) laminotomy ( $n=642)$, (2) hemilaminectomy $(n=196)$, (3) laminectomy $(n=230)$, and (4) laminectomy combined with an IF $(n=108)$ (Table 1).

The data on 1,176 patients came from 25 centres. Laminotomy was performed in 18, hemilaminectomy in 18, laminectomy in 13 and laminotomy combined with IF in 16 centres. The contribution of six centres to the study was less than five cases. The average contribution of the remaining 19 centres to the study was 61.3 cases.

Outcome measures and statistical analysis

Each patient's last available follow-up examination between 3 and 24 months postoperative was considered in the analyses. Comparison of pain relief and COMI score improvement between baseline and follow-up was performed in each group using the Wilcoxon signed-rank test.

As clinical dichotomized outcomes, achievement of minimum relevant change (MRC) in (a) COMI back and (b) leg pain and (c) COMI score of 2.2 points on the GPS [16], (d) surgical and (e) general complications, (f) measures taken due to complications, and $(\mathrm{g})$ reintervention on the index level based on patient information (COMI) were considered.

The inverse probability of treatment weighting (IPTW) using the propensity score was applied to balance the treatment groups for their baseline characteristics. IPTW using the propensity score uses weights based on the propensity score to create a synthetic sample in which the distribution of measured baseline covariates is independent of treatment assignment. The propensity score was estimated without regard to outcome variables, using multiple logistic regression analysis. The following covariates were included in the propensity score: patient age (continuous) and gender (male/female), segment (Th12/L1, L1/2, L2/3,
L3/4, L4/5, L5/S1), ASA status (1/2/3), number of previous surgeries $(0 / 1 />1)$, extent of lesion $(1 />1$ levels $)$, coexistence of additional spinal pathology (yes/no), degenerative spondylolisthesis grade I (yes/no), preop back (continuous) and leg pain (continuous), and COMI score (continuous) as well as performance of discectomy (yes/no). Univariate comparison of patient characteristics in the treatment groups before and after weighting adjustment was performed using general linear modelling or a Chi-square test as appropriate. Pairwise comparisons of back and leg pain relief and COMI score improvement was performed using general linear modelling with Bonferroni adjustments.

All statistical analyses were conducted using SAS 9.4 (SAS Institute, Inc., Cary, NC, USA). $\alpha<0.05$ was used throughout the study.

\section{Results}

The overall average follow-up interval was 12 months (range 3-24 months; on average 13 months in group 1, 11 months in group 2, 12.5 months in group 3 and 12.5 months in group 4). All four treatment groups showed significant back and leg pain relief and COMI score improvement $(p<0.001)$ between baseline and follow-up.

Propensity score-based weights were successfully calculated in the multivariate logistic regression. Table 2 shows patient characteristics before and after weighting adjustment. Before adjustment patients were significantly different in at least two of the treatment groups in all covariates except in proportions of most severely affected levels $(p=0.051)$, extent of disease $(p=0.11)$, and in preoperative COMI score $(p=0.083)$. After weighting adjustment none of the treatment groups differed from either group (Table 2).

The weighted MRC of back pain was 45.0, 34.4, 39.8, and $47.2 \%$; of leg pain 57.6, 39.4, 53.7, and 72.3\%; and of COMI score 60.9, 48.0, 59.2, and $75.3 \%$, respectively, for the four groups. Figure 1 shows how the sole decompression groups perform similarly, particularly with regard to the various degrees of back pain relief. The curves of laminectomy in combination with IF are slightly shifted to the right reflecting the achievement of MRC for pain and COMI score in a slightly higher proportion of patients.

Table 3 demonstrates the expectable (weighted) postoperative back and leg pain relief and COMI score improvement in an average patient. The weighted back pain relief was not significantly different between the treatment groups in pairwise comparisons $(p>0.10)$. The weighted leg pain relief was significantly higher after laminectomy in combination with IF in comparison with hemilaminectomy $(p<0.001)$, laminotomy $(p=0.002)$ and laminectomy $(p=0.001)$. The weighted leg pain relief after 


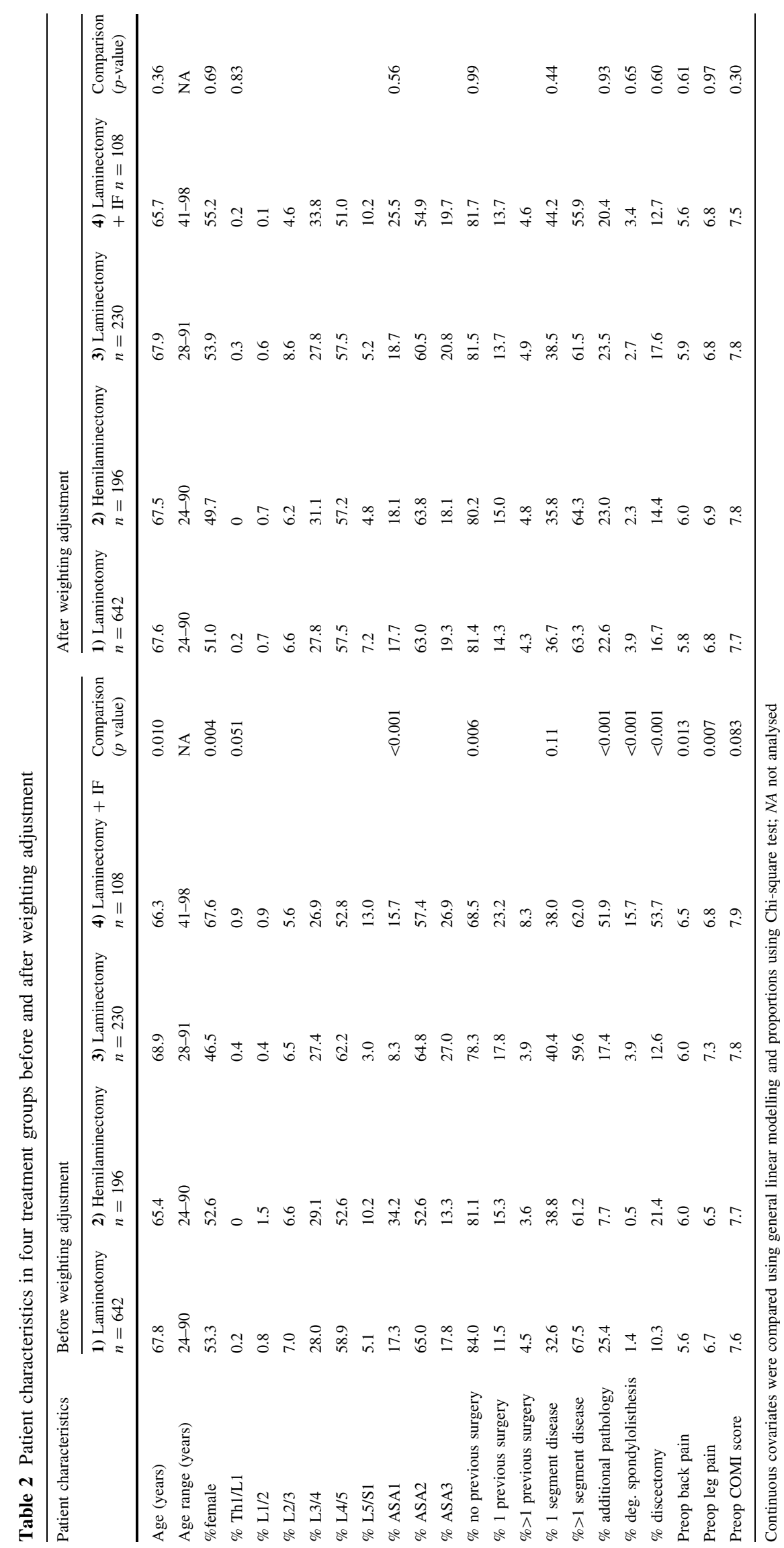


Fig. 1 The diagram

demonstrates back and leg pain relief grouped by 2-point steps between -10 (worst pain deterioration) and +10 (highest pain relief) for four treatment groups
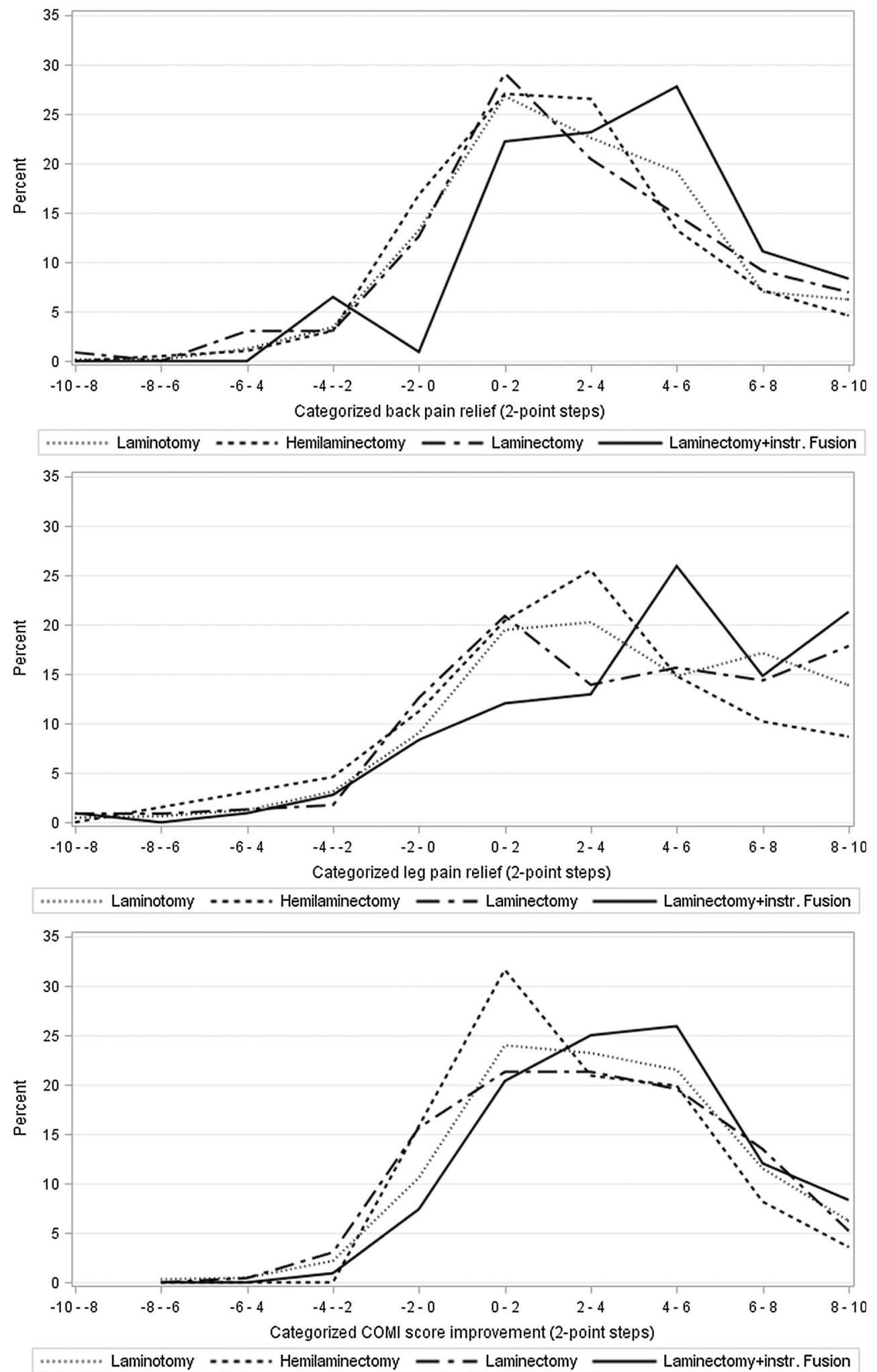

hemilaminectomy was significantly lower in comparison with laminotomy $(p=0.002)$. Regarding the weighted COMI score improvement, the hemilaminectomy group had significantly lower improvement in comparison with laminotomy ( $p=0.027)$ and laminectomy in combination with IF ( $p=0.002)$.

Surgical and general complications, measures taken and COMI-based reintervention assessments are shown in 
Table 3 Weighted

postoperative pain and pain relief with standard deviation in four treatment groups

\begin{tabular}{lllll}
\hline $\begin{array}{l}\text { Postoperative pain } \\
\text { and pain relief }\end{array}$ & $\begin{array}{l}\text { 1) Laminotomy } \\
n=642\end{array}$ & $\begin{array}{l}\text { 2) Hemilaminectomy } \\
n=196\end{array}$ & $\begin{array}{l}\text { 3) Laminectomy } \\
n=230\end{array}$ & $\begin{array}{l}\text { 4) Laminectomy + IF } \\
n=108\end{array}$ \\
\hline Back pain postop & $3.5(2.8)$ & $4.3(2.9)$ & $4.0(3.0)$ & $3.0(2.6)$ \\
Back pain relief & $2.3(3.1)$ & $1.7(2.9)$ & $1.8(3.3)$ & $2.6(2.7)$ \\
Leg pain postop & $3.6(3.2)$ & $4.8(3.1)$ & $3.9(3.5)$ & $2.1(2.4)$ \\
Leg pain relief & $3.2(3.7)$ & $2.1(3.5)$ & $2.9(4.3)$ & $4.6(3.9)$ \\
COMI score postop & $4.6(2.9)$ & $5.3(2.8)$ & $4.8(2.8)$ & $3.8(2.8)$ \\
$\begin{array}{l}\text { COMI score } \\
\text { improvement }\end{array}$ & $3.2(2.9)$ & $2.5(2.6)$ & $3.0(3.0)$ & $3.7(2.6)$ \\
\hline
\end{tabular}

Table 4. Overall, the surgical complication rates were comparable between the groups, with a slightly lower rate in the hemilaminectomy group. The overall proportions of general complications were higher in the laminectomy groups, particularly for laminectomy combined with IF. Accordingly, also the overall proportions of measures taken due to a complication were clearly higher in the laminectomy groups. The overall proportions of a reintervention as indicated by the patient on the COMI follow-up were comparable between the groups being slightly higher before adjustment in laminotomy and simple laminectomy groups.

The IPTW analysis showed that hemilaminectomy was significantly less beneficial regarding minimum relevant back pain relief in comparison with the laminectomy in combination with IF group (OR 0.59, $95 \%$ CI 0.37-0.94; Fig. 2). Laminotomy, hemilaminectomy and laminectomy were significantly less beneficial than laminectomy in combination with IF regarding leg pain (OR 0.52, $95 \% \mathrm{CI}$ 0.34-0.81; OR 0.25 , $95 \%$ CI $0.15-0.41$; OR 0.44 , $95 \%$ CI 0.27-0.72, respectively) and COMI score improvement (OR 0.51, 95\% CI 0.33-0.81; OR 0.30, $95 \%$ CI 0.18-0.51; OR 0.48, $95 \%$ CI 0.29-0.79, respectively; Fig. 2).

The likelihood of a surgical complication was significantly lower in the three decompression groups in comparison with the laminectomy combined with IF group (OR 0.42, $95 \%$ CI 0.26-0.69; OR 0.33, $95 \%$ CI 0.17-0.63; OR $0.39,95 \%$ CI $0.21-0.71$, respectively; Fig. 2). The likelihood of general complications was also significantly lower after laminotomy (OR 0.11, $95 \%$ CI 0.04-0.29), hemilaminectomy (OR $0.03,95 \% \mathrm{CI} \quad 0.003-0.41)$ and after laminectomy (OR 0.25, $95 \%$ CI 0.09-0.71), in comparison with laminectomy with IF (Fig. 2). Accordingly, the likelihood of required measures was also significantly lower after laminotomy (OR 0.28, $95 \% \mathrm{CI}$ 0.17-0.46), hemilaminectomy (OR $0.28, \quad 95 \% \mathrm{CI}$ 0.15-0.53) and after laminectomy (OR 0.39, $95 \% \mathrm{CI}$ 0.22-0.68) in comparison with laminectomy with IF (Fig. 2). The likelihood of a reintervention (indicated on COMI follow-ups) was not significantly different between the treatment groups.

\section{Discussion}

The trend for surgical treatment of LSS is steadily growing worldwide. A $45 \%$ increase for decompression and a $60 \%$ increase for fusion surgery were observed between 2004 and 2009 in the US [17]. However, the existing knowledge on comparative effectiveness of the different decompressive approaches in LSS is sparse [12].

Postacchini et al. [18] compared multiple laminotomy with laminectomy. This trial had several confounding factors [12, 18] and did not reveal any differences in clinical outcomes or spondylolisthesis progression between the two treatment methods (OR 0.85; $95 \%$ CI 0.25-2.88). Thomas et al. [19] compared outcomes of 14 patients with laminotomies and 12 with laminectomies. The comparison showed a slightly higher reduction of back, overall leg, and walking pain, and a slightly higher proportion of outcomes categorized as "good" in the latter group [19]. In an 8-year follow-up study Rompe et al. [20] were able to follow-up $61.6 \%$ of their patients and to show that simple undercutting decompression was slightly better regarding goodto-excellent outcomes than the groups with laminectomy and laminectomy in combination with IF. However, patients with laminectomy and IF were 5.1 and $6.2 \%$ more satisfied than patients with simple undercutting or laminectomy [20]. Fu et al. [21] reported laminoforaminotomy being significantly more beneficial than laminectomy at an average of 40 months after surgery with regard to back and leg pain and ODI score, although postoperatively both groups were practically free of pain and had an Oswestry score near zero. According to the Cochrane review by Gibson and Waddell, three relatively old trials considered whether some form of postero-lateral fusion, with or without instrumentation, was a useful adjunct to decompression alone (Herkowitz 1991, Bridwell 1993, Grob 1995). Pooling the three trials, a total of 139 participants with $99 \%$ follow-up at 2-3 years showed no statistically significant difference in outcomes between decompression plus fusion or decompression alone (OR 0.44; $95 \%$ CI $0.13-1.48$ ) as rated by the surgeon $18-24$ months postoperatively [12]. 
Table 4 Surgical and general complications in the groups

\begin{tabular}{|c|c|c|c|c|}
\hline $\begin{array}{l}\text { Surgical and general complications, } \\
\text { measures taken and COMI } \\
\text { follow-up-based re-intervention rates }\end{array}$ & $\begin{array}{l}\text { 1) Laminotomy } \\
n=642\end{array}$ & $\begin{array}{l}\text { 2) Hemilaminectomy } \\
n=196\end{array}$ & $\begin{array}{l}\text { 3) Laminectomy } \\
n=230\end{array}$ & $\begin{array}{l}\text { 4) Laminectomy }+ \text { IF } \\
n=108\end{array}$ \\
\hline \multicolumn{5}{|l|}{ Surgical complications } \\
\hline Wrong level (\%) & $2(3.12)$ & - & - & - \\
\hline Nerve root damage $(\%)$ & $4(6.23)$ & $2(1.02)$ & $1(0.43)$ & - \\
\hline Cauda equina damage (\%) & $3(4.67)$ & $1(0.51)$ & $1(0.43)$ & - \\
\hline Bleeding in spinal canal (\%) & $15(2.34)$ & $1(0.51)$ & $4(1.74)$ & - \\
\hline Bleeding outside spinal canal (\%) & $1(0.16)$ & $1(0.51)$ & - & - \\
\hline Malposition of implant (\%) & - & - & - & $2(1.85)$ \\
\hline Dural lesion $(\%)$ & $63(9.81)$ & $11(5.61)$ & $20(8.70)$ & $7(6.48)$ \\
\hline Wound infection (\%) & $3(0.47)$ & - & $4(1.74)$ & $2(1.85)$ \\
\hline Other $(\%)$ & $3(0.47)$ & $1(0.51)$ & $1(0.43)$ & $2(1.85)$ \\
\hline Overall patient based (\%) & $78(12.15)$ & $17(8.67)$ & $29(12.61)$ & $13(12.04)$ \\
\hline Overall patient-based rates after weighting & 11.42 & 9.20 & 10.63 & 23.50 \\
\hline \multicolumn{5}{|l|}{ General complications } \\
\hline Anaesthesiological (\%) & $1(0.16)$ & - & $2(0.87)$ & - \\
\hline Cardiovascular $(\%)$ & - & $1(0.51)$ & - & $2(1.85)$ \\
\hline Pulmonary (\%) & - & - & $2(0.87)$ & $1(0.93)$ \\
\hline Kidney/urinary (\%) & $3(0.47)$ & - & $2(0.87)$ & $2(1.85)$ \\
\hline Liver/GI (\%) & $2(0.31)$ & - & - & $1(0.93)$ \\
\hline Other $(\%)$ & $2(0.31)$ & - & - & $3(2.78)$ \\
\hline Overall patient based (\%) & $7(1.09)$ & $1(0.51)$ & $6(2.61)$ & $7(6.48)$ \\
\hline Overall patient based rates after weighting & 1.12 & 0.33 & 2.48 & 9.24 \\
\hline \multicolumn{5}{|l|}{ Measures taken } \\
\hline None* $(\%)$ & $18(21.43)$ & - & - & - \\
\hline Intervention during or after surgery* $(\%)$ & $56(66.67)$ & $13(72.22)$ & $21(60.00)$ & $8(50.00)$ \\
\hline Conservative* $(\%)$ & $7(8.33)$ & $4(22.22)$ & $8(22.86)$ & $6(37.50)$ \\
\hline Extended hospital stay* $(\%)$ & $12(14.29)$ & $1(5.56)$ & $6(17.14)$ & $7(43.75)$ \\
\hline Overall patient based (\%) & $64(9.97)$ & $18(9.18)$ & $33(14.35)$ & $15(13.89)$ \\
\hline Overall patient-based rates after weighting & 9.51 & 9.54 & 12.64 & 27.22 \\
\hline \multicolumn{5}{|c|}{ Reintervention on the index level endorsed by patients in COMI follow-ups } \\
\hline Reinterventions (\%) & $17(2.65)$ & $2(1.02)$ & $6(2.61)$ & $1(0.93)$ \\
\hline Reinterventions after weighting (\%) & 2.90 & 0.84 & 1.84 & 3.25 \\
\hline
\end{tabular}

"Other" surgical complications in group 1 were fat tissue necrosis, wound dehiscence and instability of one segment; in group 2, persistent low back pain; in group 3, bladder dysfunction; and in group 4, neurapraxia of peroneal nerve and ulnar nerve palsy. The two specified "other" general complications in group 4) were damages from the patient position on the surgical table and MRSA sepsis. One "other" general complication in group 4 and both "other" general complications in group 1 were not further specified. * the denominator for the percentages was the number of patients with a surgical or/and general complication in the groups (e.g. 84, 18, 35, and 16, respectively)

This paucity of evidence led us to perform the current study based on the international spine registry Spine Tango. To account for potential selection bias in a voluntary registry, we applied a propensity score based, inverse probability of treatment method. Four main propensity score methods can be distinguished: propensity score matching, stratification on the propensity score, IPWT using the propensity score, and covariate adjustment using the propensity score [22]. The analysis of a propensity score-matched sample can mimic that of a randomized controlled trial (RCT) [22]. This method is recommended for large studies with sufficient matching pairs. When comparing our four treatment groups, a simple pair match of 1:1:1:1 would require an even larger sample size. On the other hand, in some settings propensity score matching and IPTW remove systematic differences between studied subject groups to a comparable degree [22]. Finally, matching results in a selection of practically the same 
Fig. 2 Plot of odds ratios with $95 \%$ CI for laminotomy (1), hemilaminectomy (2), and laminectomy (3) in comparison with laminectomy with IF (instrumented fusion) (4) for each of seven outcomes. Note: for the outcomes $a-c$ the odds ratios with confidence intervals below the reference line demonstrate inferior results; for the outcomes $d-g$ the odds ratios with confidence intervals below the reference line demonstrate superior results

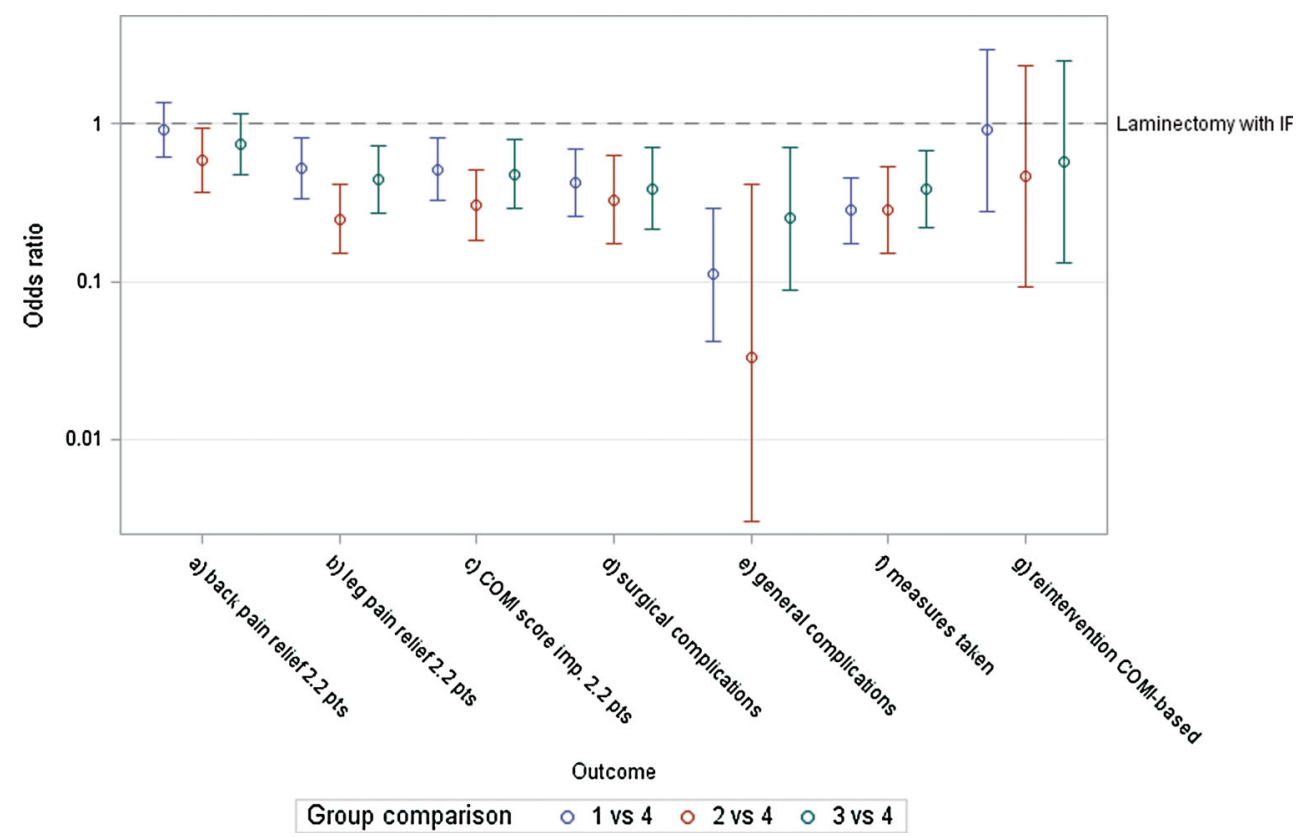

patients from each treatment group and exclusion of other, differing cases, which itself introduces a selection bias into the more or less daily practice-based patient populations. We chose the IPTW method for our study, and good weighting was achieved. Without clear clinical guidelines and indications for one or the other treatment procedure, such an adjustment for patient baseline characteristics was the only option for our comparative study of the four procedures, in which patients with similar characteristics and probabilities underwent either of the treatments.

Laminectomy with IF was clearly more beneficial than either decompression method alone with respect to the probability of achieving minimum relevant leg pain relief and COMI score improvement of 2.2 points. Improvement in COMI score can be equated with an increased overall function, as the score is calculated based on pain, backrelated function, symptom-specific well-being, general quality of life and disability domains. However, surgical and general complications occurred significantly more frequently in the arthrodesis patients. Wider confidence intervals were seen for general complication estimatesparticularly for hemilaminectomy, which was due to the low number of events. Potentially explained by the higher rate of complications, also the likelihood for measures taken were significantly higher in the arthrodesis patients in comparison with the decompressed patients. The hemilaminectomy group had the lowest probability of desired back and leg pain relief and COMI score improvement, although the complication rates were also the lowest in this group. No significant differences were seen in proportions of reinterventions endorsed by the patients in COMI follow-ups.
The average follow-up time in the study was 12 months, though an invasive treatment such as decompression in combination with an IF ideally should be followed up longer for an accurate judgement of patient benefits. In the short term, the more invasive approach appears advantageous regarding patient-related outcomes. It also appears superior regarding probability of early reoperation as reported by Deyo et al. [23]. The reoperation probability was higher for the decompression group over the first year but was lower than that for the simple and complex arthrodesis groups after 2 years. In other words, after 2 years the probability of having another operation is higher for patients with an arthrodesis.

Longer term data on LSS are not yet available in the Spine Tango registry. Long-term studies in the literature suggest high patient satisfaction and good clinical results after laminotomies (limited evidence study) [21], laminectomies (limited evidence studies) [24-26], and after laminectomies with IF (level I study) [27]. Potentially different perioperative treatment costs favouring sole decompression groups in our comparison can be expected, but were not part of the analysis. In the trends study of Bae et al. [17], fusions were associated with a longer length of stay and hospital costs greater than decompression charges by a multiple of three to four times.

Finally, IF is a technically more complex and invasive procedure for which particular surgical skills are required and longer surgery time and more complications can be expected. These factors should be considered when deciding the optimal approach in treating LSS. Although a significantly higher proportion of patients treated with IF have achieved an MRC in leg pain and a desirable COMI 
score, the question should be raised whether these results mean that laminectomy with IF should be the method of choice in LSS considering the higher probability of surgical and general complications. The other side of the coin is showing that the proportion of patients with desirable pain and COMI score outcomes in the sole decompression groups is lower than in the fusion group, but not low. Technically simpler decompressive surgery should not be unreasonably supplemented by an IF. The addition of fusion with or without instrumentation to surgical decompression is completely justified when decompression alone leads to spinal instability. However, there are no guidelines to help define situations when the risk is large enough to justify the increased complexity, cost, and complications in patients with spinal stenosis. In a level $\mathrm{V}$ review (expert opinion), Atlas et al. noted in summary that “... given the increased complications associated with fusion procedures, decompressive laminectomy should be the surgical procedure of choice for most patients who have not had previous spine surgery or who do not have other concomitant conditions such as degenerative spondylolisthesis" [8, 12]. According to our results, the studied decompression procedures, with different levels of invasiveness, should not have the same expected outcomes. A hemilaminectomy appears as less beneficial in comparison with other decompression types. Decompression alone can be later on extended into a fusion, but a fusion can less easily be reversed into a simple decompression. Hence, decompression types which the study identified as the most beneficial ones (laminotomy, laminectomy) are probably the therapies of choice, also from a cost-benefit ratio point of view.

\section{Limitations}

Some limitations of this study require mention. The accuracy of complication reporting in a voluntary registry cannot be estimated. Documentation of complications and all other data in the Spine Tango registry relies on individual surgeons' and hospitals' honesty. $65.1 \%$ of the data used in the analysis came from centres whose reliability has already been assessed and confirmed several times, but the reliability of the centres associated with the remaining data has not been assessed previously. However, no hospitals involved in the analysis are known to be unreliable. A good indicator of honest documentation is the proportion of dural lesions. The proportions of reported dural lesions we observed, of 9.8, 5.6, 8.7 and $6.5 \%$ in the respective groups, were comparable with those seen in the national SWEspine registry for patients with decompressive surgery $(8.5 \%)$ and patients with decompressive surgery + fusion for LSS $(5.5 \%)$ [28]. The rates for dural lesions in our study (overall rate $8.8 \%$ ) were even higher than those observed in the study by Stromqvist et al. [28] (overall rate $7.4 \%$ ), which suggests credible reporting. Even if Spine Tango complication rates might potentially be underreported, we have no reason to believe that the underreporting would affect the treatment groups to a different extent and thus bias the comparative analysis. A Spine Tango code of conduct will be introduced in 2014 to foster honest, transparent, and monitored documentation.

As mentioned above, various measurable and unmeasurable factors may influence surgical decision making in favour of one approach or another [29]. We could adjust for the most relevant covariates but one may also have to include body mass index, smoking habits, and presence of psychosocial factors (flags). However, these data could not be recorded in the 'surgery 2006' forms. They are part of the new 'surgery 2011' form and may be considered in future analyses.

The analysis did not include physician-based followups, which would allow assessing the physician documented reintervention rates. As already mentioned above, long-term follow-up analyses are required, where detailed reintervention rates must be considered.

The numbers of general complications and reinterventions endorsed by patients were relatively low in the groups. Caution is required when interpreting rare events as they may disproportionally impact the overall picture.

When comparing patient outcomes after different treatments using MRC, one should be aware that MRC may differ for different treatment measures. We based the analysis on the 2.2 points reported as the MRC for the graphical COMI scales [16]. The MRC in the literature ranges between 1 and 4.5 points for various 0 to 10 rating scales and 2 points is an often proposed cut-off [30].

The reader may agree that it would be nearly impossible to set up an RCT comparing four different decompression techniques. Moreover, RCT results have high internal but low external validity in contrast to limited internal but high external validity of observational effectiveness studies. A large registry like Spine Tango represents an optimal data pool for comparative effectiveness analyses using appropriate statistical methods.

\section{Conclusions}

Our large study of prospectively documented patients demonstrates that back and leg pain associated with LSS significantly improve after all decompression procedures. None of the studied treatment options appears definitively superior to another. However, based on the results laminotomy or laminectomy is somewhat more recommendable than hemilaminectomy for minimum relevant pain relief. 
Despite better patient outcomes after laminectomy in combination with instrumented fusion, caution is advised due to higher rates of surgical and general complications and consequent required measures. An objective assessment of spinal instability and guidelines for the addition of instrumented fusion are needed.

Acknowledgments The participants of the Spine Tango Register are acknowledged for their continuous contribution that makes possible such studies reflecting the daily practice of spine surgeons. The data of the following centres were used (in alphabetic order of country, city, hospital and department): Dept. of Spinal Surgery in Royal Adelaide Hospital (Australia); Dept. of Spinal Surgery in St. Andrew's Hospital in Adelaide (Australia); Dept. of Orthopaedic Surgery in University Hospital of Graz (Austria); Dept. of Orthopaedic Surgery in Edith Cavell Clinic of Brussels (Belgium); Dept. of Orthopaedic Surgery in Saint Pierre Clinic of Ottignies (Belgium); Dept. of Neurosurgery in Köpenick Hospital DRK Kliniken Berlin (Germany); Dept. of Orthopaedic Surgery in University Hospital of Cologne (Germany); Dept. of Neurosurgery in Hospital CologneMerheim (Germany); Dept. of Spine Surgery in Hospital Dortmund (Germany); Dept. Of Orthopaedic Surgery in University Hospital of Greifswald (Germany); Group Practice of Orthopaedics and Neurosurgery in Hof (Germany); Dept. of Special Spine Surgery in Leopoldina Hospital of Schweinfurt (Germany); Dept. of Neurosurgery in Sapienza University of Rome (Italy); Dept. of Orthopaedic Surgery in Tan Tock Seng Hospital (Singapore); Dept. of Orthopaedic Surgery in University Hospital of Ljubljana (Slovenia); Dept. of Neurosurgery in Bethesda Hospital of Basel (Switzerland); Dept. of Spine Surgery in Bethesda Hospital of Basel (Switzerland); Dept. of Orthopaedic Surgery in Salem Hospital of Bern (Switzerland); Dept. of Spine Surgery in The Spine Center Thun (Switzerland); Dept. of Orthopaedic Surgery in Hospital Schwyz (Switzerland); Dept. of Orthopaedic Surgery in Zollikerberg Hospital (Switzerland); Dept. of Spine Surgery in University Hospital Balgrist of Zurich (Switzerland); Spine Unit of Nuffield Oxford Centre (UK); Dept. of Neurosurgery in Walton Centre Foundation Trust of Liverpool (UK); Division of Spine Surgery in NYU Hospital of New York (USA).

Conflict of interest None.

\section{References}

1. Hart LG, Deyo RA, Cherkin DC (1995) Physician office visits for low back pain. Frequency, clinical evaluation, and treatment patterns from a U.S. national survey. Spine 20:11-19

2. Schamie N (2011) Lumbar spinal stenosis: The growing epidemic. AAOSNow. http://www.aaos.org/news/aaosnow/may11/ clinical10.asp. Accessed May 2014

3. Deyo RA (2010) Treatment of lumbar spinal stenosis: a balancing act. Spine J 10:625-627

4. Kovacs FM, Urrutia G, Alarcon JD (2011) Surgery versus conservative treatment for symptomatic lumbar spinal stenosis: a systematic review of randomized controlled trials. Spine 36:E1335-E1351

5. Weinstein JN, Tosteson TD, Lurie JD, Tosteson AN, Blood E, Hanscom B, Herkowitz H, Cammisa F, Albert T, Boden SD, Hilibrand A, Goldberg H, Berven S, An H, Investigators S (2008) Surgical versus nonsurgical therapy for lumbar spinal stenosis. N Engl J Med 358:794-810

6. Malmivaara A, Slatis P, Heliovaara M, Sainio P, Kinnunen H, Kankare J, Dalin-Hirvonen N, Seitsalo S, Herno A, Kortekangas
P, Niinimaki T, Ronty H, Tallroth K, Turunen V, Knekt P, Harkanen T, Hurri H, Finnish Lumbar Spinal Research Group (2007) Surgical or nonoperative treatment for lumbar spinal stenosis? A randomized controlled trial. Spine 32:1-8

7. Atlas SJ, Keller RB, Robson D, Deyo RA, Singer DE (2000) Surgical and nonsurgical management of lumbar spinal stenosis: four-year outcomes from the maine lumbar spine study. Spine 25:556-562

8. Atlas SJ, Delitto A (2006) Spinal stenosis: surgical versus nonsurgical treatment. Clin Orthop Relat Res 443:198-207

9. Weinstein JN, Lurie JD, Olson PR, Bronner KK, Fisher ES (2006) United States' trends and regional variations in lumbar spine surgery: 1992-2003. Spine 31:2707-2714

10. Irwin ZN, Hilibrand A, Gustavel M, McLain R, Shaffer W, Myers M, Glaser J, Hart RA (2005) Variation in surgical decision making for degenerative spinal disorders. Part I: lumbar spine. Spine 30:2208-2213

11. Katz JN, Lipson SJ, Lew RA, Grobler LJ, Weinstein JN, Brick GW, Fossel AH, Liang MH (1997) Lumbar laminectomy alone or with instrumented or noninstrumented arthrodesis in degenerative lumbar spinal stenosis. Patient selection, costs, and surgical outcomes. Spine 22:1123-1131

12. Gibson JN, Waddell G (2005) Surgery for degenerative lumbar spondylosis. Cochrane database Syst Rev 2005(4):CD001352

13. EuroSpine (2013) Spine Tango. http://www.eurospine.org/spinetango.htm. Accessed May 2014

14. Rosenbaum PR, Roubin DB (1983) The central role of the propensity score in observational studies for casual effects. Biometrika 70(1):41-45

15. Carreon LY, Glassman SD, Kantamneni NR, Mugavin MO, Djurasovic M (2010) Clinical outcomes after posterolateral lumbar fusion in workers' compensation patients: a case-control study. Spine 35:1812-1817

16. Mannion AF, Porchet F, Kleinstuck FS, Lattig F, Jeszenszky D, Bartanusz V, Dvorak J, Grob D (2009) The quality of spine surgery from the patient's perspective: part 2. Minimal clinically important difference for improvement and deterioration as measured with the Core Outcome Measures Index. Eur Spine J 18(3):374-379

17. Bae HW, Rajaee SS, Kanim LE (2013) Nationwide trends in the surgical management of lumbar spinal stenosis. Spine 38:916-926

18. Postacchini F, Cinotti G, Perugia D, Gumina S (1993) The surgical treatment of central lumbar stenosis. Multiple laminotomy compared with total laminectomy. JBJS Br 75:386-392

19. Thomas NW, Rea GL, Pikul BK, Mervis LJ, Irsik R, McGregor JM (1997) Quantitative outcome and radiographic comparisons between laminectomy and laminotomy in the treatment of acquired lumbar stenosis. Neurosurgery 41:567-574 (discussion 574-565)

20. Rompe JD, Eysel P, Zollner J, Nafe B, Heine J (1999) Degenerative lumbar spinal stenosis. Long-term results after undercutting decompression compared with decompressive laminectomy alone or with instrumented fusion. Neurosurg Rev 22:102-106

21. Fu YS, Zeng BF, Xu JG (2008) Long-term outcomes of two different decompressive techniques for lumbar spinal stenosis. Spine 33:514-518

22. Austin PC (2011) An introduction to propensity score methods for reducing the effects of confounding in observational studies. Multivar Behav Res 46:399-424

23. Deyo RA, Martin BI, Kreuter W, Jarvik JG, Angier H, Mirza SK (2011) Revision surgery following operations for lumbar stenosis. JBJS Am 93:1979-1986

24. Bouras T, Stranjalis G, Loufardaki M, Sourtzis I, Stavrinou LC, Sakas DE (2010) Predictors of long-term outcome in an elderly group after laminectomy for lumbar stenosis. J Neurosurg Spine 13:329-334 
25. Iguchi T, Kurihara A, Nakayama J, Sato K, Kurosaka M, Yamasaki K (2000) Minimum 10-year outcome of decompressive laminectomy for degenerative lumbar spinal stenosis. Spine 25:1754-1759

26. Wilby MJ, Seeley H, Laing RJ (2006) Laminectomy for lumbar canal stenosis: a safe and effective treatment. Br J Neurosurg 20:391-395

27. Slatis P, Malmivaara A, Heliovaara M, Sainio P, Herno A, Kankare J, Seitsalo S, Tallroth K, Turunen V, Knekt P, Hurri H (2011) Long-term results of surgery for lumbar spinal stenosis: a randomised controlled trial. Eur Spine J 20:1174-1181

28. Stromqvist F, Jonsson B, Stromqvist B, Swedish Society of Spinal Surgeons (2012) Dural lesions in decompression for lumbar spinal stenosis: incidence, risk factors and effect on outcome. Eur Spine J 21:825-828

29. Sobottke R, Aghayev E, Roder C, Eysel P, Delank SK, Zweig T (2012) Predictors of surgical, general and follow-up complications in lumbar spinal stenosis relative to patient age as emerged from the Spine Tango Registry. Eur Spine J 21:411-417

30. Ostelo RW, Deyo RA, Stratford P, Waddell G, Croft P, Von Korff M, Bouter LM, de Vet HC (2008) Interpreting change scores for pain and functional status in low back pain: towards international consensus regarding minimal important change. Spine 33:90-94 